\title{
Une ontologie du temps pour le langage naturel
}

\author{
Françoise GAYRAL, Philippe GRANDEMANGE \\ Laboratoire d'Informatique de l'université Paris-Nord (LIPN) \\ Institut Galilée, avenue Jean-Baptiste Clément, 93430 Villetaneuse (France)
}

\section{ENGLISH ABSTRACT}

We propose a new ontology for the time in natural language which provides the following features :

- it renders an account of most of the temporal phenomena of language (dates, duration, events, states); it offers capacities for the comprehension of the narratives ;

- in the contrary to the traditional systems, it needs no hard "types" for dealing with the different classes used by the terminology : thus an event may date others "when John died, there were many demonstrations in the world", a date may be the begiming of an cvent "monday, Paul was leaving for a six months tour" ; our ontology endures the fluidity of natural language which does not make rigid, in the narratives, the signification of the temporal entities ;

- it allows some multiplicity of points of vien (partially inconsistent) about a single event : in the sentence "the travel of Christophe Colomb, which endured a long time, has been the beginning of a rich period of exploration", "the voyage of $\mathrm{CC}^{\circ}$ is seen as a simple event in the main proposition and as a complex event in the subordinate one.

With respect to these issues, the ontology uses the following frameworks:

- a KLONE-like network aiming at a quick detection of incoherences : it contains taxonomic inferences (including the whole terminology) plus the facts; a bulk of rules (assertional device) embodying contingent properties ;

- non-monotonic reasoning is needed to revise simplistic conclusions obtained from superficial descriprions : the rules and the links of the network may be default rules;

- the VaDe (Variable Depth) system supports our ontology ; it is implemented with an ATMSlike truth maintenance system.

The flexible ontology we present offers an interesting frame for further researches in computational linguistics, in particular in the domain of the interpretation of the narratives. 


\section{Introduction}

Nous proposons une ontologie temporelle adaptée au traitement des différents phénomènes temporels apparaissant dans des énoncés en langage naturel. Il s'agit de rendre compte des événements, de leur durée, de leur localisation et aussi des relations entre ces événements, qu'elles soient purement temporelles (d'inclusion, de précédence...) ou qu'eltes correspondent à des liens de structuration (relation d'un événement à ses sousévénements) ou de causalité. Il est important de noter que la langue est très souple dans l'utilisation qu'elle permet des entités temporelles, notamment dans les rôles variés qu'elle leur fait jouer : un événement peut servir de date pour un autre (á la mort de son père. Paul déménagea), il peut exprimer la durée d'un autre (pendant tout le voyage, Paul a lu), il peut avoir un début qui est une date (Paul a commencé à travailler à 14 heures), un événement peut être perçu comme atomique (le voyage de Christophe Colomb fut le début d'une riche période d'explorations) ou au contraire comme décomposable (le voyage de Christophe Colomb fiat long et pénible : après avoir fait face à plusieurs tempêtes, l'équipage dut affronter une épidémie de typhus). Un événement peut même, à l'intérieur d'un seul récit, être considéré de plusieurs points de vue partiellement incompatibles (le voyage de Christophe Colomb qui dura de longs mois fut le début d' une riche période d' explorations qui fait percevoir le voyage comme atomique dans la principale el structurél dans la relative).

Notre ontologie temporelle doit donc être suffisamment riche et souple pour capter cette capacité de la langue à accepter une représentation non figée des entités temporelles [Kayser 90). Cette souplesse de l'ontologie implique la possibilité de révision du raisonnement parce que la suite du récit peut imposer une autre interprétation que celle qui avait été adoptée jusque là, par exemple lorsque des informations plus précises sont fournies. Elle s'appuie done sur une logique nonmonotone : la logique des défauts.

\footnotetext{
'Les termes "complexe", "structurê", "décomposiable".. rendent compte de la mênte idóc intuitivs.
}

Une fois précisés nos objectifs, nous montrons pourquoi les différentes représentations du temps proposées en IA sont inadéquates (section 2); la section 3 présente nos choix de base à travers la description rapide de quelques classes de l'ontologie tandis que la section suivante présente quelques règles précisant la signification des entités utilisées. La section 5 traite d'une propriété particulière de l'ontologie : l'adaptabilité à la variation des points de vue en compréhension de récits.

\section{Temps et langage naturel}

Peu de systèmes de représentation et de raisonnement temporel allient la prise en compte de toutes les facettes du temps dans la langue et des changements de perspective imposés par les récits.

Les logiques temporelles classiques [Prior 57] permettent seulement de "temporaliser" les propositions en les situant dans un axe passé/futur grâce aux opérateurs ${ }^{2} \mathrm{H}$ et $\mathrm{G}$ et à leurs duaux mais aucune information métrique (il y a trois jours, elle est allée a Nancy) ni d'inclusion temporelle (pendant que Paul dormait, Virginie prépara le repas) ne peut être codée.

Allen [Allen 81] choisit d'associer a tout événement un intervalle (celui sur lequel il a lieu) et de traduire toute information temporelle entre deux événements par des relations temporelles entre leurs intervalles associés. Il exhibe treize relations de base, exclusives les unes des autres; mais la discrimination entre ces relations (notamment 'before' et 'meets') oblige à regarder la position des bornes de chacun des intervalles, ce qui est très délicat dans la langue : le cas le plus courant est de ne pas savoir situer exactement les intervalles, notamment au niveau de leurs bornes. De plus, ce système permet difficilement de traiter de concepts métriques, comme la durée d'un événement, ou d'ancrage des événements dans le temps notamment par la prise en compte de dates ou de références temporelles.

McDemott [McDermott 82] au contraire sépare fort judicieusement une ontologie strictement temporelle (la droite réelle des dates) et une ontologie plus "événementielle", la relation entre

${ }^{2}$ Si p est unc proposition, Hp signifie 'il a toujours été le cas que $p$ ', $G p$ 'il sera toujurs le cas que $p$ '. 
les deux n'intervenant que ponctuellement grâce à sa fonction de datation. C'est sa définition de l'événement (ensemble d'intervalles d'état) qui ne convient pas au tratement du langage naturel où elle doit, selon nous, être primitive.

Kamp [Kamp 81 ] choisit justement de baser sa représentation du temps sur les concepts d'événement et d'état. Mais les règles de constructions de ses DRS (Discourse Representation Structure) sont décevantes dans la mesure où elles amènent à un grain fixxe de représentation et à une perspective toujours identique: aux verbes au passé simple correspondent des événements, aux verbes à l'imparfait des états...

\section{Propositions pour une ontologie}

L'ontologie temporelle que nous proposons s'appuie sur des réflexions linguisticues et se concrétise non seulement dans la définition d'un réseau sémantique [Brachman Schmolze 85] à la KL-ONE (figure 1) qui offe une terminologie aux notions temporelles repérables de la langue mais encore dans des règles qui donnent leur sémantique aux classes et aux rôles. Pour plus de clarté, nous présentons d'abord en détail le réseau puis quelques règles considérées comme représentatives parmi les 92 existant dans le système.

Notre choix fondanental consiste d'une part à considérer les événements comme les primitives de base de notre représentation et d'autre part à ne pas avoir de représentation explicite du temps "réel"? usuellement implanté en termes d'instants ou d'intervalles. En effet, il n'est pas de récit sans événement ; par contre il y a récit même sans appel au temps réel. Celuici n'est pas pour autant éliminé, il intervient couramment pour repérer des événements par leur date (le 4 Juillet 1980), pour exprimer une durée (pendant trois heures), par l'expression d'une fréquence (tous les lundis) ou d'un écart (cing minutes plus tard).

Nous distinguons parmi toutes les notions temporelles ${ }^{4}$ exprimées dans la langue par

\footnotetext{
3 I.c temps réel est assimilé couramment à un axe orienté de gauche à droite el graduable suivant des unitén prédéfinies pour lesquelles il existe des règles de conversion.

4 Nous laisserons de côté, pour l'instam, le temps des verbes.
}

différents marqueurs ${ }^{5}$ deux grandes catégories [Borillo 86] :

--> celles qui permettent de localiser un événement : y sont attachées toutes les références aux notions de précédence, simultanéité, inclusion... Toute information de ce type donne une réponse à la question 'quaud l'événement a-t-il lieu?'.

-> celles qui permettent d'exprimer une durée, repérées par le fait qu'elles permettent de répondre aux questions du type 'depuis combien de temps?', 'pendant combien de temps ?'...

Nous traduisons ces deux catégories par l'existence de deux classes : la classe Ref Temp (pour référence temporelle) qui concrétise toute information de type localisation, la classe Laps pour toute information de type durée.

Un objet de type Laps peut désigner soit la durée d'un événement ou plus généralement d'une référence temporelle (il a travaillé pendant trois heures) soit un laps de temps écoulé entre deux événements (ll a pris sa moto. Dix minutes plus tard, il s'est fait renverser). Dans le premier cas, on écrira une relation entre la référence temporelle et sa durée grâce au rôle apourpuree; dans le second cas, on définira le laps par ses bornes et ce sont les rôles commence et termineA qui seront remplis ainsi éventuellement que le rôle valnumLaps.

Pour préciser la classe Reftemp, il est nécessaire d'analyser dans la langue la manière dont sont localisés les événements. Un événement peut être situé grâce à un complément de date, grâce à des adverbes de liaison, voire même grâce à la simple séquentialité des phrases dans le récit qui implique (par défaut) la succession des événements qu'elles évoquent.

Lorsque l'événement est lexicalisé par un verbe conjugué, le temps du verbe suffit à placer l'événement par rappont au temps d'énonciation dans le cas des temps simples (passé ou futur), par rapport à d'autres événements dans le cas

\footnotetext{
5 Que ce soicnt des mots, des expressions ou même des propositions subordonnées de temps.
} 


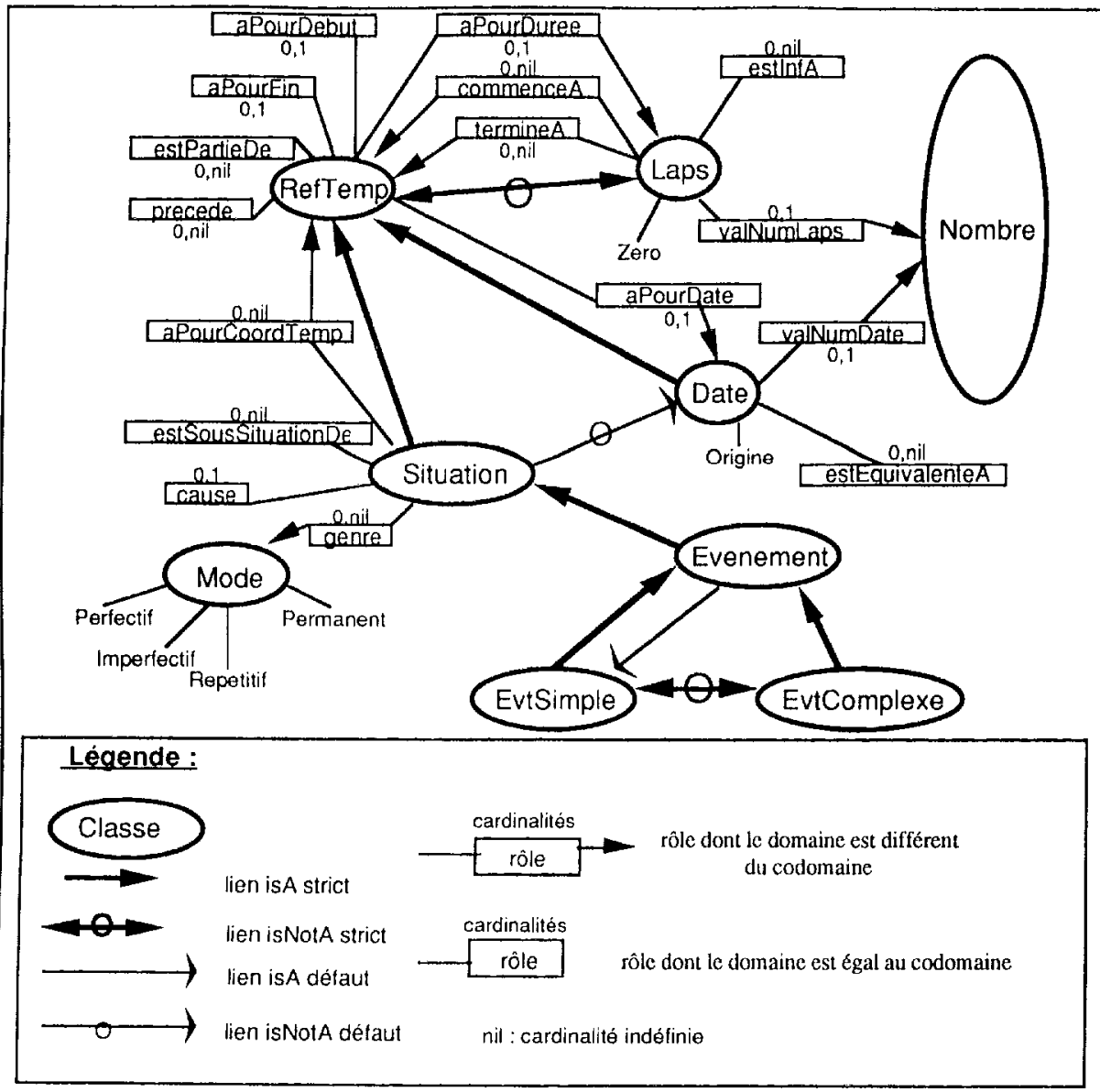

Remarque : les relations de préordrc (precede, estPartieDe, estinfA) sont cntendues au sens large.

Figure 1 : le réseau de l'ontologie temporelle

des temps composés (plus que parfait, futur antérieur etc.) |Reichenbach 47|.

Dans le cas d'un complément de date, la localisation d'un événement peut s'établir :

--> par rappon au monent d'énonciation dans le cas d'adverbiaux déictiques: hier, la semaine prochaine.

--> par rapport atu point de référence courant (déjà établi) dans le cas d'adverbiaux anaphoriques: la veille, à ce moment là.
$->>$ de manière absolue. Leur fixation sur l'axe du temps est, dans ce cas, connue ${ }^{6}: \grave{a} 14$ heures, le 14 Janvier 1980.

--> en faisant référence à d'autres événements ou situations : $a$ la mort de Louis XVI, pendant la construction de Beaubourg, a la mort de grand-pere, lors de son stage, quand tu travaillais...

On s'aperçoit ainsi qu'on peut classer les références temporelles (objets de la classe

${ }^{6}$ Serait-ce de façon vague pour sa mesure. 
Ref Temp) en deux classes : la classe pate qui recouvre les trois premiers cas et la classe Situation qui recouvre le dernier. Dans tous les cas, cette idée de localisation d'un événement (ou situation) se concrétise par le rôle apourcoordtemp de codomaine Ref Temp. Un objet de type Date est un marqueur tempore] qui n'est pas a priori un événement et qui permet, en théorie, une projection sur l'axe du temps. Une date peut être absolue : sa fixation sur laxe du temps est, dans ce cas, entièrement connue (en Août 1914, le 14 Janvier 1980) ou peut en tout cas être restituée (l'emploi de à 14 heures ou de pendant le mois de Janvier sousentend que le jour (ou l'année) est déjà connu). Les adverbiaux déictiques tels que hier, la semaine prochaine sont considérés comme des dates puisqu'un calcul temporel pemet de leur faire correspondre un repère sur l'axe du temps objectif. Les adverbiaux anaphoriques comme la veille, à ce moment là, dont la référence est supposée résolue, font également partie des dates.

La classe situation regroupe les notions d'événement et d'état. Aucune classe ne correspond à la notion d'état dans notre ontologie?.

Parmi les objets de type situation, nous distinguons les événements, définis explicitement par l'existence de la classe Evenement et du lien is A entre Evenement et Situation .

La classe Evenement est la classe de base de notre système de représentation; elle joue donc le rôle de classe pivot en organisant les autres classes par différence. Les événements peuvent être lexicalisés aussi bien par des verbes que par des noms : l'orage de dimanche dernier, le voyage de Christophe Colomb son des événements. Pour permetre de rendre compte des différentes caractéristiques d'un événement, nous créons deux sous-classes (incompatibles) de la classe Evenement : Evtsimple et EvtComplexe dont nous parlerons plus en détail dans le paragraphe 5 .

7 La discrimination entre unc situation qui n'est pats un événement (un étal) el un événement n’obíil pas à des critères facilement repérables dans une plirasc. En cils de doute, on acerochera à la classe situation toul objet s'inscrivant dans le temps cui n'est pas une date ou une durcéc.
Dans le réseau de la figure 1 , il est important de noter que les classes Date et Laps sont plus proches du temps réel que les classes RefTemp, Situation, Evenement. En effet, des valeurs numériques peuvent leur être associées et des calculs permettent d'assurer une certaine consistance aux raisonnements faits. On vérifiera par exemple systématiquement qu'un laps a une durée compatible avec ses dates de début et fin.

Dans les rôles attachés aux différentes classes, nous distinguons les rôles temporels comme precede, est partiede des rôles de structuration comme aPourDebut et estsoussituationde. Les faits du type estsoussituationde $(a, b)$ peuvent correspondre soit à des connaissances stéréotypées (reconnues grâce à des scripts, qui sont normalement inclus dans le lexique), soit à la structure même du récit dans la manière dont sont présentés les événements 8 .

\section{Un exemple : les relations entre un événement et sa coordonnée temporelle}

Nous faisons l'hypothèse que comprendre un récit n'est pas reconstituer dans son ensemble une réalité, c'est percevoir les relations principales entre les événement évoqués : les règles que nous utilisons tentent d'en rendre compte. Il s'agit soit de règles strictes notées '-->', soit' de règles de défaut notées '--)', défauts normaux dont la justification ne sera pas explicitée puisqu'elle coïncide avec la conclusion. Nous omettons dans la suite les quantificateurs universels qui régissent les variables de ces règles.

Dans cette section, nous nous intéressons plus particulièrement aux relations entre un événement et sa coordonnée temporelle en vue d'obtenir une structuration des entités temporelles du récit.

\footnotetext{
${ }^{8}$ En particulier les techniques de mise en perspective des ćvénements: plus de détails à ce sujet dans [Gayral 92]. Par cxemple, la phrase lors de son siage, Paul a renconiré Virginie propose la rencontre comme sous événement du stage.

${ }^{9}$ Le système dispose également de règles concluant à l'existence d'objets. Nous ne les donnons pas ici.
} 
** Nous considérons que quand la coordonnée temporelle d'un événement est une date, on peut seulement déduire un rapport temporel d'inclusion entre l'événement et sa date.

- Sans information sur les durées de ces deux objets, on considérera que la coordonnée temporelle est un "nid" pour l'événement qui se trouve ainsi temporellement inclus dans sa date. C'est le rôle de la règle 84 :

84. Evenement $(x) \wedge$ apourCoordTemp $(x, c x)$ $\rightarrow$ estpartiede $(x, c x)$

Cette règle peut être illustrée par dimanche, il y' a eu un orage où l'on conclura à l'inclusion temporelle de l'orage dans la journée de dimanche.

- Lorsqu'on "en sait plus", notamment dins les durées relatives de l'événement et de sa date, précisément dans le cas où la durée de la date est connue et est inférieure à la durée connue de l'événement qu'elle réfère, on conclut que cette date est à la fois le début de l'événement et sa date par lat règle 86.

86. Evenement $(x) \wedge$ Date $(c x) \wedge$

$$
\begin{aligned}
& \text { apourcoordTemp }(x, c x) \wedge \\
& \text { apourduree }(x, d u x) \wedge \\
& \text { apourduree }(c x, d u c x) \wedge \\
& \text { estinfA (ducx, dux) }--)
\end{aligned}
$$

aPourDate $(x, c x) \wedge$ aPourDebut $(x, c x)$

Des exemples peuvent être : vers 14 heures Paul a téléphoné longtemps à Virginie ou dimanche, Paul est parti en voyage pour hait jours ${ }^{10}$.

\footnotetext{
10 Les exemples concernent des procès qui peuvent se décomposer en au moins une partic initiale el unc autre. Pour le premier exemple, téléphoner peut se décomposer en au moins deux procès : appeler au lélćphone el communiquer, on peut donc considérer qu'il existe une autre formulation équivalente : vers 14 heures, $P$ aul a appeli Virginie el ils ont bavardé longtemps ; dans ce cas, il y a glissement de sens du verbe vers son début et la coordonnéc temporelle est celle non pas du process tout entier mais celle de son début. De même dans le deuxième exemple, le procès correspondant au syntagme partir en voyage peut en réalité se décomposer en deux procès (le départ el le voyage lui-même). La durée explicitéc porte sur le voyage alors que la coordonnéc temporelle est celle du départ.

Nous ne décomposons ni dans un cats ni dans l'autre ces procès mais nous déduisons des relations temporelles les concernant globalement. Il s'agil bien de traiter des glissements de sens sur les verbes, mais pas par choix dans un ensemble (supposé totalement ćnuméré) do "glissements possibles".
}

*** Lorsque cette coordonnée temporelle est une situation, nous pensons que la mise en rapport de ces situations par le récit n'est pas "innocente" et traduit le plus souvent une relation sémantique entre elles. Cette relation sémantique est signifiée par le rôle estSoussituationDe qui indiquera aussi par défaut une relation d'inclusion temporelle. Nous écrivons ainsi des règles qui vont permettre de créer de toutes pièces ces relations. Ce sont bien sûr des règles de défaut car il est des cas où le locuteur voulait simplement signifier un rapport temporel entre les situations.

D'abord, exposons deux de ces règles qui, comme pour les dates, vont distinguer le cas général où on ne sait rien de très précis dans les rapports de durée entre la situation et sa coordonnée temporelle et les cas où au contraire on dispose de données objectives.

-- Le cas général est traité par la règle 83 :

$$
\begin{aligned}
& \text { 83. Evenement }(x) \wedge \text { Situation }(\mathrm{cx}) \wedge \\
& \text { aPourCoordTemp }(x, \mathrm{cx}) \\
& --) \text { estSoussituationDe }(x, c x)
\end{aligned}
$$

On peut l'illustrer par lors de mon stage, $j$ 'ai rencontré Marie.

- Lorsque la coordonnée temporelle d'un événement est une situation de durée inférieure à celle de l'événement qu'elle réfère, on conclut d'une part qu'elle en est le début et d'autre part qu'elle en est une sous-situation grâce à la règle 81.

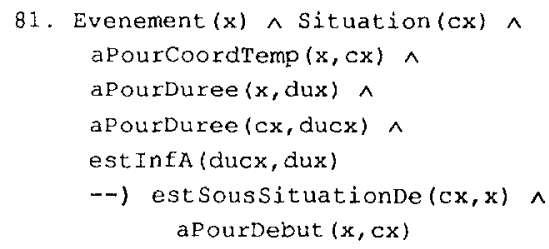

L'exemple qui peut être donné est le suivant: A la mort de son père qui fut foudroyante, Paul a plongé dans une déprime de plusieurs mois.

\section{Objectifs de l'ontologie}

L'ontologie que nous proposons ne suppose pas, contrairement à celles basées sur des logiques monotones, l'établissement de preuves solides de consistance pour opérer sur elle. Nous faisons l'hypothèse qu'une telle situation modélise le raisonnement fait par l'auditeur d'un récit: il n'a nul besoin de tout savoir ou de considérer que tout est cohérent pour 
comprendre, c'est à dire pour extraire des informations simples sur ce que son interlocuteur a voulu dire.

Ainsi, une de nos préoccupations consiste en l'adaptation constante de la représentation d'un événement au récit où il s'insère. Cette capacité d'adaptation de la représentation d'un même événement a différentes conséquences. En premier lieu, nous choisissons de focaliser en partie le raisonnement sur un choix dans une alternative : un événement est simple (vision globale) ou complexe (vision analyticue). Ainsi, un événement complexe est muni de nombreux attributs que n'a pas a priori un événement simple : sa durée, son début, sa fin, des sousévénements... En second lieu, nous considérons que, sans indication particulière, un événement est considéré comme simple, de manière à être fidèle à une minimalité de la représentation.

L'appartenance d'un événement à l'une ou l'autre des deux classes Evtsimple et EvtComplexe ne correspond à aucune réalité : un événement n'est pas simple ou complexe en soi. Son "accrochage" à l'une des deux classes ne traduit qu'un point de vue local et provisoire imposé par le récit : c'est uniquement par rapport aux autres événements et au déroulement des méandres du récit que "l'importance" d'un événement peut être évaluée.

Un événement peut être vu comme complexe dans deux sortes de situarions : d'une part si on lui découvre un sous-événement, d'autre part si des informations venant de l'extérieur remplissent des attributs de cet événement (par exemple, la durée) :

73. Evenement $(x) \wedge$ apourduree $(x$, dux $) \wedge$ $\neg=($ dux, zero $) \rightarrow$ Evtcomplexe $(x)$

Dans d'autres cas, un événement a priori complexe doit pouvoir êtue vu comme simple (règle 71): dans le long et pénible voyage de Christophe Colomb fut le début d'une riche période d'explorations le voyage de Christophe Colomb est vu comme simple puisqu'il marque le début de la riche période d'explorations."

11 Cette phrase, formuléc difićremment pourrail amener à la conclusion inverse : c'est parce qu'ici l'accent est mis par le "récil" sur la riche période d'explorations qu'une telle conchusion peut ètre tiréc. Ceci ouvre une piste de recherche qui nécessite l'sudte approfondie des
71

Evenement (ssx) $\wedge$ Evenement $(x) \wedge$ estSoussituationie (ss $x, x)$

-) Evtsimple(ssx)

\section{Conclusion}

Notre proposition rompt avec la tradition des logicues temporelles en plaçant la notion d'événement au cour de l'analyse, en attachant au temps "objectif" le simple rôle de vérificateur de consistance d'une déduction non monotone, en préservant la possibilité d'une pluralité des significations... L'ontologie proposée rend compte de la grande souplesse de la langue quant aux rôles joués par les différentes entités temporelles les unes par rapport aux autres. Elle permet également de traiter des changements de perspectives dans les récits qui sont peu ou mal abordés par les raisonneurs sémantiques traditionnels, même s'il reste à approfondir l'étude des marqueurs linguistiques permettant de repérer dans le cours d'un récit ces changements de perspectives.

Notre ontologie enfin est intégrée dans un système implémenté (VaDe). Basé sur une conception à profondeur variable de la représentation et du raisonnement, VaDe [Grandemange 92] est un système hybride, doté d'un module de maintenance de la cohérence, permettant de manipuler de façon originale les formules existentielles.

Nous tenons à remercier Daniel Kayser et Christophe Fouqueré pour leur lecture attentive et leurs pertinentes remarques.

\section{Bibliographie}

[Allen 81] James F. ALLEN : An intervalbased representation of temporal knowledge ; in Proc. of the $7^{\text {th }}$ IJCAI, p. $221 \mathrm{sq}$, Vancouver (BC, Canada), 1981.

\section{[Borillo 86] Andrée BORILLO : Les emplois adverbiaux des noms de temps ; dans Actes du séminaire "Lexique et Traitement Automatique des Langages", pp. 11-36, université Paul Sabatier, Toulouse (F), 16-17 janvier 1986.}

marcueurs de la langue qui permettent de distinguer, dans le récit, un événement par rapport à d’autres [Gayral 92]. 
[Brachman Schmolze 85] Ronald J.

BRACHMAN, James G. SCHMOLZE :

An overview of the Kl-one

knowledge representation system;

in Cognitive Science 9(2):171-216, 1985.

[Gayral Grandemange 91] Françoise

GAYRAL, Philippe GRANDEMANGE :

Événements : ponctualité et

durativité ; dans Actes du $8^{e ̀ n e}$ congrès

RFIA (AFCET-INRIA), Lyon-

Villeurbanne (F), 27-29 novembre 1991.

[Gayral 92] Françoise GAYRAL:

Sémantique du langage naturel et profondeur variable : une première approche; thèse de l'université de Paris-Nord, Villetaneuse ( $F$ ), janvier 1992.

[Grandemange 92] Philippe GRANDEMANGE : Représentation des connaissances et profondeur variable : une implantation; thèse de l'université de Paris-Nord, Villetaneuse (F), février 1992.
[Kamp 81] Hans KAMP : Événéments, représentations discursives et référence temporelle, Langages, 64:34-64, 1981 .

[Kayser 90] Daniel KAYSER : Truth and the interpretation of natural language : a non-monotonic variabledepth approach ; in Proc. of the $9^{\text {th }}$ ECAI, pp. 392-397, Stockholm (Sweden), august 6-10 1990 [(L. C. Aiello ed.) Pitman, London (UK)].

[McDermott 82] Drew V. MCDERMOTT : A temporal logic for reasonning about processes and plans ; Cognitive Science 6:101-155, 1982.

[Prior 57] A. N. PRIOR : Time and modality, Clarendon Press, Oxford (UK), 1957.

[Reichenbach 47] H. REICHENBACH : Elements of symbolic logic, McMillan, New York (USA), 1947. 